\title{
Infrarotspektrum, Elektronen-Absorptionsspektrum und HMO-Rechnung 4.5-Dioxo-2-thioxo-1.3-dithiolan $\mathrm{C}_{3} \mathrm{O}_{2} \mathrm{~S}_{3}$
}

\author{
A. Müller, B. Krebs * und R. Ahlrichs ** \\ Anorganisch-Chemisches Institut und Lehrstuhl für Theoretische Chemie der Universität Göttingen \\ (Z. Naturforschg. 21 b, 389-393 [1966] ; eingegangen am 4. Februar 1966) \\ The infrared and electron absorption spectra of 4.5-dioxo-2-thioxo-1.3-dithiolane are reported and \\ discussed. Using the HMO method, $\pi$ electron densities, $\pi$ bond orders, the transition energy \\ $E_{\mathrm{N}} \rightarrow \mathrm{V}_{1}$ and the total $\pi$ electron energy are calculated.
}

Kürzlich berichteten KREBS und GatTow ${ }^{1}$ über die Darstellung 4.5-Dioxo-2-thioxo-1.3-dithiolan $\mathrm{C}_{3} \mathrm{O}_{2} \mathrm{~S}_{3}$ aus Trithiokohlensäure und Oxalylchlorid. In der vorliegen Arbeit sollen weitere Eigenschaften, insbesondere die Bindungsverhältnisse dieser interessanten, in weinroten Nadeln kristallisierenden Verbindung untersucht werden.

Der Bindungszustand des $\mathrm{C}_{3} \mathrm{O}_{2} \mathrm{~S}_{3}$-Moleküls kann in der Sprache der Methode der Valenzstrukturen durch folgende, am wahren Zustand wahrscheinlich mit größtem Gewicht beteiligten Grenzstrukturen wiedergegeben werden:<smiles>O=C1SC(=S)SC1=O</smiles>

(I)<smiles>O=C1OC(=O)C(=S)S1</smiles>

(II)<smiles>Oc1oc(=S)oc1O</smiles>

(II)<smiles>O=c1oc(=O)c(=O)c1=O</smiles>

(II)<smiles>O=C1SC(=O)C(=O)S1</smiles>

(ए)
Da sich aus dem Schwingungsspektrum der Verbindung Aussagen über die Bindungsverhältnisse gewinnen lassen, haben wir das IR-Spektrum näher

\begin{tabular}{|c|c|}
\hline Bandenlagen $\left[\mathrm{cm}^{-1}\right]$ & Zuordnung \\
\hline $1722(\mathrm{st})$ & $v(\mathrm{CO})$ \\
$1520 ?(\mathrm{~s})$ & $v(\mathrm{C}=\mathrm{S})$ \\
$1106(\mathrm{st})$ & \\
$1000(\mathrm{~m})$ & \\
$955(\mathrm{~m})$ & \\
$(925)(\mathrm{Sch}) ?^{\mathrm{a}}$ & \\
$(845)(\mathrm{s}) ?^{\mathrm{a}}$ & hauptsächlich in der $\mathrm{CS}_{\mathrm{I}}\left(\mathrm{S}_{\mathrm{II}}\right)_{2^{-}}$ \\
$520(\mathrm{~m})$ & Gruppe lokalisiert \\
$463(\mathrm{~m})$ & Gruppis \\
\hline
\end{tabular}

Tab. 1. Absorptionsmaxima im IR-Spektrum von $\mathrm{C}_{3} \mathrm{O}_{2} \mathrm{~S}_{3}$ (in Nujol) im Bereich zwischen 4000 und $420 \mathrm{~cm}^{-1}$. a Möglichkeit der Überlagerung schwacher Banden mit Banden vom Nujol.

untersucht. Die ermittelten Absorptionsbanden gehen aus Abb. 1 und Tab. 1 hervor.

Die Bande mit starker Intensität bei $1722 \mathrm{~cm}^{-1}$ ist der $v(\mathrm{CO})$-Schwingung zuzuordnen. Bei Kopplung der beiden Carbonylschwingungen sind eigentlich zwei Absorptionsbanden zu erwarten, nämlich die einer Intakt- und die einer Gegentaktschwingung. Abgesehen von der Andeutung einer Schulter sind

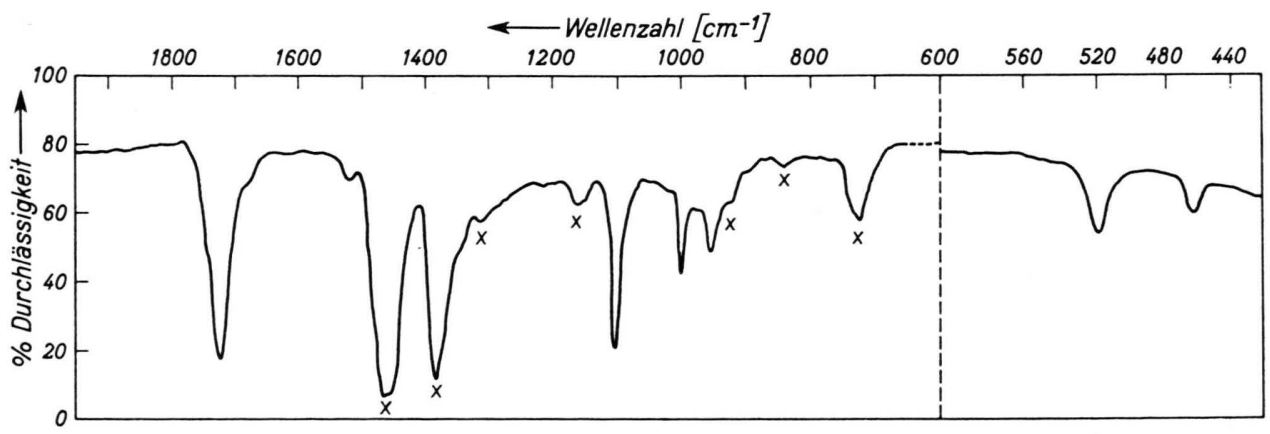

Abb. 1. IR-Spektrum von $\mathrm{C}_{3} \mathrm{O}_{2} \mathrm{~S}_{3}$ (in Nujol; $\mathrm{X}$ : Nujolbanden).

* Zur Zeit: Brookhaven National Laboratory, Upton, L. I., N. Y., USA.

** Lehrstuhl für Theoretische Chemie.
1 B. Krebs u. G. Gatrow, Angew. Chem. 75, 978 [1963] ; Angew. Chem. Intern. Ed. 2, 618 [1963]. 
jedoch Anzeichen für die Aufspaltung der $v(\mathrm{CO})$ Bande im IR-Spektrum nicht zu finden. Die (z. B. im Vergleich zum Cyclopentanon) relativ niedrige Frequenz der $v(\mathrm{CO})$-Schwingung ist durch das relativ größere Gewicht zu erklären, mit dem die Grenzstrukturen mit „aufgerichteten $\pi$-Bindungen“ am

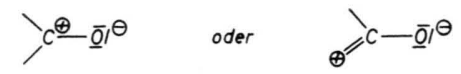

Bindungszustand der CO-Gruppierungen beteiligt sind. Ein Anteil der Strukturen (II) und (III) (vgl. oben) am wahren Bindungszustand des Moleküls unter Einbeziehung der CO-Gruppen in das gesamte $\pi$-Bindungssystem mußte zu einer Erniedrigung der Frequenz von $v(\mathrm{CO})$ durch Erniedrigung der CO. Valenzkraftkonstanten bzw. des entsprechenden Bindungsgrades führen. Hierbei ist allerdings zu beachten, daß die Kraftkonstanten der hier betrachteten Bindungen nicht allein durch verschiedene $\pi$-Bindungsgrade, sondern ebenfalls durch Änderungen in den Hybridisierungs-Verhältnissen der $\sigma$-Bindungen beeinflußt werden. Dieser Effekt ist besonders bei kleinen Ringen wesentlich, wie der Vergleich der Frequenzen von $v(\mathrm{CO})$ folgender Ketone zeigt: Cyclobutanon (fl.) $1782 \mathrm{~cm}^{-12}$, Cyclopentanon (fl.) $1742 \mathrm{~cm}^{-13,4}$, Cyclohexanon (fl.) $1714 \mathrm{~cm}^{-13}$.

Eine grobe Abschätzung nach Siebert ${ }^{5}$ unter $\mathrm{Zu}$ grundelegung des Zweimassenmodelles zur Berechnung von $f_{\mathrm{CO}}$ und ohne Berücksichtigung der $\sigma$-Hybridisierung ergibt für den CO-Bindungsgrad im $\mathrm{C}_{3} \mathrm{O}_{2} \mathrm{~S}_{3}$ einen Wert von

$$
N_{\mathrm{CO}}=1,9 \text {. }
$$

Die starke Bande bei $1106 \mathrm{~cm}^{-1} \mathrm{kann}$ mit großer Wahrscheinlichkeit einer Schwingung zugeordnet werden, die hauptsächlich in der Thiocarbonylbindung lokalisiert ist. Die Frequenz der $\mathrm{C}=\mathrm{S}$ Valenzschwingung liegt bei ähnlichen bisher spektroskopisch untersuchten Verbindung im Bereich etwa zwischen 1200 und $1050 \mathrm{~cm}^{-1}$; die Absorptionsbande ist im allgemeinen sehr intensiv (vgl. l. c. ${ }^{4}$ ). Die Möglichkeit, daß die schwächere Bande bei $1000 \mathrm{~cm}^{-1}$ dieser Schwingung zuzuordnen ist, kann wegen ihrer sehr viel geringeren Intensität praktisch ausgeschlossen werden.

${ }^{2}$ K. Frei u. H. H. Günthard, J. Mol. Spectroscopy 5, 218 [1960].

3 E. J. Hartwell, R. E. Richards u. H. W. Thompson, J. chem. Soc. [London] 1948, 1436.

4 R. Mecke, R. Mecke u. A. Lütrringhaus, Chem. Ber. 90, 975 [1957].

5 H. Siebert, Z. anorg. allg. Chem. 273, 170 [1953].
Bei allen anderen Banden (einschließlich der Bande bei $1000 \mathrm{~cm}^{-1}$ ) handelt es sich um die Absorptionen von Gerüstschwingungen des Fünferringes und um Deformationsschwingungen der CO- und CS-Gruppen, wobei, wie die Auswahlregeln für eine angenommene $\mathrm{C}_{2 \mathrm{v}}$-Symmetrie (ebene Struktur) des Moleküls zeigen, bei der verwendeten Probekonzentration nicht alle IR-aktiven Schwingungen beobachtet worden sind. Die Lage der Banden bei 520 und $463 \mathrm{~cm}^{-1}$ verdient besondere Erwähnung. In allen bisher von uns spektroskopisch untersuchten Verbindungen mit einer Gruppierung

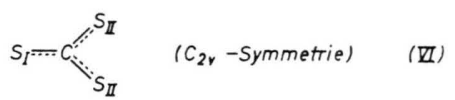

sind im Frequenzbereich zwischen 550 und $450 \mathrm{~cm}^{-1}$ zwei Absorptionsbanden vorhanden, die im Trithiocarbonat-Ion $\mathrm{CS}_{3}{ }^{2 \ominus} \quad\left(\mathrm{D}_{3 \mathrm{~h}}\right.$-Symmetrie $)$ der totalsymmetrischen Valenzschwingung $v_{1}\left(\mathrm{~A}_{1}{ }^{\prime}\right)$ und der nichtebenen $\mathrm{CS}_{3}$-Deformationsschwingung $v_{2}\left(\mathrm{~A}_{2}{ }^{\prime \prime}\right)$ entsprechen (vgl. Tab. 2). Dies läßt vermuten, daß

\begin{tabular}{|c|c|c|}
\hline & Bandenlagen $\left[\mathrm{cm}^{-1}\right]$ & Literatur \\
\hline $\mathrm{BaCS}_{3}$ & $510 \quad(\mathrm{R}), \quad 516$ & $6 \mathrm{~b} \quad 6 \mathrm{c}$ \\
\hline $\mathrm{Ni}\left(\mathrm{NH}_{3}\right)_{3} \mathrm{CS}_{3}$ & $505, \quad 490$ & $6 \mathrm{~d}$ \\
\hline $\mathrm{Zn}\left(\mathrm{NH}_{3}\right)_{2} \mathrm{CS}_{3}$ & 519,494 & $6 \mathrm{~d}$ \\
\hline $\mathrm{Co}_{2}\left(\mathrm{NH}_{3}\right)_{6} \mathrm{C}_{2} \mathrm{~S}_{7}$ & 510,483 & $6 \mathrm{e}$ \\
\hline$\left(\mathrm{NH}_{4}\right)_{2} \mathrm{CS}_{4}$ & $519, \quad 485$ & unveröffentlicht \\
\hline $\mathrm{SC}(\mathrm{SH})_{2}$ & 485,465 & $6 f$ \\
\hline $\mathrm{C}_{3} \mathrm{O}_{2} \mathrm{~S}_{3}$ & 520,463 & diese Arbeit, 1 \\
\hline
\end{tabular}

Tab. 2. IR-Absorptionsmaxima im Bereich zwischen 550 und $450 \mathrm{~cm}^{-1}$ von Verbindungen mit einer $\mathrm{CS}_{\mathrm{I}}\left(\mathrm{S}_{\mathrm{II}}\right)_{2}$-Gruppierung.

bei der Einbeziehung der Gruppe (VI) in den hier vorliegenden fünfgliedrigen Ring die Banden bei 520 und $463 \mathrm{~cm}^{-1}$ Schwingungen zuzuordnen sind, die hauptsächlich in der $\mathrm{CS}_{\mathrm{I}}\left(\mathrm{S}_{\mathrm{II}}\right)_{2}$-Gruppierung lokalisiert sind. Hierbei entspricht sinngemäß eine der Banden einer Schwingung, die im wesentlichen eine symmetrische $v_{\mathrm{s}}\left(\mathrm{CS}_{\mathrm{II}}\right)$-Schwingung darstellt, die andere entspricht einer nichtebenen Deformationsschwingung der Gruppe (VI). Über die relative Lage dieser beiden Schwingungen in verschiedenen Trithiocarbonaten vgl. l. c. ${ }^{6 \mathrm{a}}$. Die geringe Kopplung

${ }^{6}$ a) A. Müller u. B. Krebs, Spectrochim. Acta, im Druck; b) A. Müller u. M. Stockburger, Z. Naturforschg. 20 a, 1442 [1965] ; c) B. Krebs, G. Gattow u. A. Müller, Z. anorg. allg. Chem. 337, 278 [1965] ; d) B. Krebs u. A. MüLLER, Z. Naturforschg. 20 a, 1664 [1965]; e) A. Müller u. B. Krebs, Z. anorg. allg. Chem., im Druck; f) G. Gatrow u. B. Krebs, Z. anorg. allg. Chem. 321, 143 [1963]. 
der Schwingungen der Gruppierung (VI) mit den anderen Schwingungen des Ringes ist auf die relativ große Masse des Schwefelatoms zurückzuführen.

In Abb. 2 ist das Elektronenspektrum von $\mathrm{C}_{3} \mathrm{O}_{2} \mathrm{~S}_{3}$ im sichtbaren und UV-Bereich wiedergegeben. Das Spektrum zeigt eine schwache Doppelbande bei 522 und $540 \mathrm{~nm}$, eine weitere recht schwache Absorptionsbande bei $417 \mathrm{~nm}$ und eine sehr intensive Bande

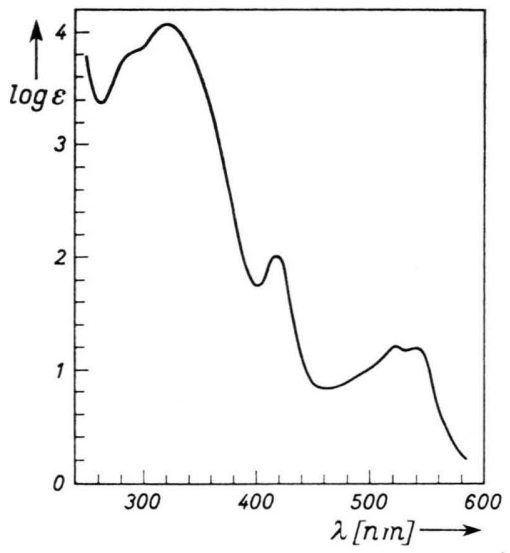

Abb. 2. Sichtbares und UV-Spektrum von $\mathrm{C}_{3} \mathrm{O}_{2} \mathrm{~S}_{3}$ (in $\mathrm{CCl}_{4}$, $10^{-3}$ bis $10^{-6}-m$. Lösungen).

bei $324 \mathrm{~nm}$ mit einer Schulter bei $290 \mathrm{~nm}$. Die Banden bei 540,522 und $417 \mathrm{~nm}$ weisen deutliche Verschiebungen der Absorptionsmaxima beim Wechsel des Lösungsmittels auf (vgl. Tab. 3). Stärker polare Lösungsmittel wie Methanol oder Äthanol konnten nicht verwandt werden, da hier leicht Zersetzung der Substanz eintritt.

\begin{tabular}{|c|c|c|}
\hline \multirow{2}{*}{ Lösungsmittel } & \multicolumn{2}{|c|}{ Absorptionsmaxima [nm] } \\
\hline & Bande $1+2$ & Bande 3 \\
\hline $\begin{array}{l}\text { n-Hexan, } \mathrm{CCl}_{4} \\
\text { Benzol } \\
\text { Diäthyläther } \\
\text { Dioxan }\end{array}$ & $\begin{array}{l}540, \quad 522 \\
530 \text { (nicht aufgelöst) } \\
516 \text { (nicht aufgelöst) } \\
510\end{array}$ & $\begin{array}{c}417 \\
\text { Schulter bei } 415 \\
411 \\
\text { Schulter bei } 405\end{array}$ \\
\hline
\end{tabular}

Tab. 3. Abhängigkeit der Lage der $n \rightarrow \pi^{*}$-Banden des $\mathrm{C}_{3} \mathrm{O}_{2} \mathrm{~S}_{3}$ vom Lösungsmittel.

Die Doppelbande im längstwelligen Teil des Spektrums ist auf Grund ihrer Lage, ihrer Intensität und ihrem Verhalten gegenüber dem Lösungsmittel

7 J. W. Sidman, Chem. Reviews 58, 689 [1958].

${ }^{8}$ C. N. R. Rao, Ultraviolet and Visible Spectroscopy, Butterworth, London 1961.

9 M. J. Janssen, Receuil Trav. chim. Pays-Bas 79, 454, 464, 1066 [1960].

10 J. Fabian u. R. Mayer, Spectrochim. Acta 20, 299 [1964]. (hypsochrome Verschiebung mit zunehmender Polarität des Lösungsmittels) einem $n \rightarrow \pi^{*}\left({ }^{1} \mathrm{U}-{ }^{1} \mathrm{~A}\right)$ Ubergang ${ }^{7}$ der Thiocarbonylgruppe zuzuordnen. Ein Vergleich mit $n \rightarrow \pi^{*}$-Übergängen anderer Thiocarbonyl-Verbindungen ${ }^{8-11}$ zeigt, daß die diesem Übergang entsprechende Bande im vorliegenden Fall in einem relativ langwelligen Bereich liegt. Eine Erklärung hierfür kann die teilweise Einbeziehung des bindenden $\mathrm{p}_{\tau}$-Orbitals am S-Atom in das gesamte $\pi$-Bindungssystem der Molekel sein, wodurch eine Aufrichtung der CS-Bindung ermöglicht wird. Im Thiobenzophenon, das durch ein sehr ausgedehntes $\pi$-Bindungssystem gekennzeichnet ist, liegt z. B. der entsprechende Übergang bei $600 \mathrm{~nm}^{12}$ (Messung in Äthanol). Die Aufspaltung der Bande im vorliegenden Fall des $\mathrm{C}_{3} \mathrm{O}_{2} \mathrm{~S}_{3}(540-522 \mathrm{~nm})$ ist wahrscheinlich auf eine Schwingungsfeinstruktur zurückzuführen, die in polareren Lösungsmitteln verschwindet.

Die weitere schwache Bande bei $417 \mathrm{~nm}$, die ebenfalls eine hypsochrome Verschiebung mit einer $\mathrm{Zu}$ nahme der Polarität des Lösungsmittels zeigt, soll versuchsweise einem $n \rightarrow \pi^{*}$.Übergang der Carbonylgruppe zugeordnet werden. Während für isolierte CO-Gruppen Wellenlängen von etwa $280 \mathrm{~nm}$ für den $n \rightarrow \pi^{*}$-Übergang beobachtet werden, kann durch Einbeziehung der Carbonylgruppe in ein System von konjugierten Doppelbindungen $\operatorname{der} n \rightarrow \pi^{*}$.Übergang nach längeren Wellen verschoben werden. So zeigen z. B. konjugierte Dialdehyde und Diketone eine schwache Absorption bei $450 \mathrm{~nm}^{13}$. Eine theoretisch mögliche Aufspaltung der Bande wegen der Anwesenheit zweier CO-Gruppen läßt sich nicht beobachten, da der Energieunterschied der beiden resultierenden Zustände ${ }^{1} \psi_{\mathrm{n}}{ }^{3} \pm{ }^{1}{\psi_{\mathrm{n}}}^{\prime}{ }^{13} \mathrm{zu}$ gering ist.

Auf Grund ihrer starken Intensität ist die weiterhin beobachtete Bande bei $324 \mathrm{~nm}$ (Schulter bei $290 \mathrm{~nm}$ ) einem erlaubten Elektronenübergang zuzuordnen. Die relativ langwellige Lage der Bande im Vergleich zu $\pi \rightarrow \pi^{*}$-UUbergängen in ThiocarbonylVerbindungen (vgl. z. B. l. c. ${ }^{10}$ ) weist darauf hin, daß die Bande eventuell einem Übergang $\pi \rightarrow \pi^{*}$ $\left(\mathrm{N} \rightarrow \mathrm{V}_{1}\right)$ von der letzten besetzten MO, die sich über das ganze Molekül erstreckt, zur ersten unbesetzten MO entspricht ${ }^{16}$; dabei gehen in die

11 K. Rosengren, Acta chem. scand. 16, 2284 [1962].

12 P. Brocklehurst u. A. Burawoy, Tetrahedron 10, 118 [1960].

13 J. N. Murrell, The Theory of the Electronic Spectra of Organic Molecules, Methuen, London 1963. 
$\pi$-MO's die $\pi$-Elektronen der beiden Carbonylverbindungen und der Thiocarbonylbindung, sowie die zwei $\mathrm{p}_{\pi}$-AO's (p-Modell ${ }^{14}$ ) der beiden Ringschwefelatome ein.

Da von Zahradnik und Mitarbb. ${ }^{14}$ und von Mayer und Mitarbb. ${ }^{15,16}$ gezeigt worden ist, daß sich die $\mathrm{Hückelsche} \mathrm{MO-Methode}{ }^{17}$ auf heterocyclische Schwefelverbindungen anwenden läßt und auch $\mathrm{N} \rightarrow \mathrm{V}_{1}$-Übergänge beschreiben kann, haben wir an unsere Untersuchungen über $\mathrm{C}_{3} \mathrm{O}_{2} \mathrm{~S}_{3}$ eine HMO-RechRechnung angeschlossen ${ }^{17 \mathrm{a}}$.

Für die Coulomb- ( $\alpha$ ), Resonanz- $(\beta)$ und Uberlappungsintegrale wurden die in der HMO. Methode verwandten Näherungen benutzt. Weiterhin wurden die für Heteroatome gewöhnlich verwendeten Coulomb- und Resonanzintegrale bzw. die üblicherweise als $h_{\mathrm{X}}$ und $k_{\mathrm{CX}}$ benutzten Werte übernommen ${ }^{15}: \alpha_{\mu}=\alpha+h_{\mu \beta}$ und $\beta_{\mu \nu}=k_{\mu \nu} \cdot \beta ;=0: h_{0}$ $=1,3$ und $k_{\mathrm{CO}}=1,4 ;=\mathrm{S}: h_{\mathrm{S}}=0,5$ und $k_{\mathrm{CS}}=0,9$; $-\mathrm{S}-: h_{\mathrm{S}}=1,0$ und $k_{\mathrm{CS}}=0,7$. Die Bestimmung der Eigenwerte $E_{\mathrm{i}}$ bzw. $\mathrm{H}$ ü c k el - Zahlen

$$
x=\frac{a-E}{\beta}
$$

und Eigenvektoren ( $\pi$ MO's) erfolgte durch Transformation der Energiematrix auf Diagonalgestalt nach dem Jakobi-Verfahren. Weiterhin wurde die Gesamt- $\pi$-Elektronenenergie:

$$
E=\sum_{i} n_{\mathrm{i}} x_{\mathrm{i}},
$$

die $\pi$-Elektronendichte am $\mu$-ten Atom:

$$
q_{\mu}=\sum_{i} n_{\mathrm{i}} c_{\mathrm{i} \mu}{ }^{2},
$$

die $\pi$-Bindungsordnung zwischen den Atomen $\mu$ und $\nu$ :

$$
p_{\mu \nu}=\sum_{i} n_{\mathrm{i}} c_{\mathrm{i} \mu} c_{\mathrm{i} \nu}
$$

und die Energie des Überganges $\mathrm{N} \rightarrow \mathrm{V}_{1}$ :

$$
E_{N \rightarrow V_{1}}=\left(x_{6}-x_{5}\right) \beta \text { berechnet. }
$$

In dem folgenden Moleküldiagramm sind die berechneten Größen $q_{\mu}$ und $p_{\mu v}$ zusammengefaßt (in Klammern Nettoladungen: $Q_{\mathrm{SI}_{\mathrm{I}}}=Q_{0}=1-q ; Q_{\mathrm{C}}=$ $\left.1-q ; Q_{\mathrm{SII}}=2-q\right)$.

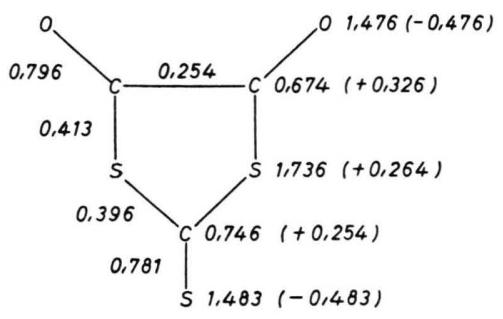

Die $\pi$-Ladungsdichten und $\pi$-Bindungsstärken haben plausible Werte. Die $8 \mathrm{H}$ ü c k e l - Zahlen $x$ ergaben sich wie folgt: $x_{1}: 2,7357 ; x_{2}: 2,0561 ; x_{3}: 1,7513$; $x_{4}: 1,0496 ; x_{5}: 0,7512 ; x_{6}:-0,3512 ; x_{7}:-1,0869$; $x_{8}:-1,8057$.

Die untersten 5 HMO's werden mit den $10 \pi$-Elektronen nach dem $\mathrm{P}$ a uli-Prinzip besetzt. Hiermit ergibt sich für den Übergang von der letzten besetzten MO zur ersten nichtbesetzten eine Übergangsenergie

$$
E=\left(x_{6}-x_{5}\right) \beta=1,1024 \beta .
$$

Die Transformationsmatrix, die die Energiematrix auf Diagonalgestalt transformiert und die als Spal-

\begin{tabular}{|l|c|r|r|r|r|r|r|r|}
\hline & \multicolumn{1}{|c|}{$x_{1}$} & \multicolumn{1}{c|}{$x_{2}$} & \multicolumn{1}{c|}{$x_{3}$} & \multicolumn{1}{c|}{$x_{4}$} & \multicolumn{1}{c|}{$x_{5}$} & \multicolumn{1}{c|}{$x_{6}$} & $x_{7}$ & \multicolumn{1}{c|}{$x_{8}$} \\
\hline$\varphi_{1}$ & 0,0587 & 0,0000 & 0,3982 & 0,0000 & $-0,7611$ & 0,2829 & $-0,4247$ & 0,0000 \\
$\varphi_{2}$ & 0,2474 & $-0,2124$ & 0,4366 & $-0,6559$ & 0,3753 & $-0,1147$ & $-0,3069$ & 0,1568 \\
$\varphi_{3}$ & 0,2474 & 0,2124 & 0,4366 & 0,6559 & 0,3753 & $-0,1147$ & $-0,3069$ & $-0,1568$ \\
$\varphi_{4}$ & 0,4559 & $-0,5934$ & $-0,2639$ & 0,2599 & $-0,2017$ & $-0,4146$ & $-0,0995$ & 0,2833 \\
$\varphi_{5}$ & 0,4559 & 0,5934 & $-0,2639$ & $-0,2599$ & $-0,2017$ & $-0,4146$ & $-0,0995$ & $-0,2833$ \\
$\varphi_{6}$ & 0,4675 & $-0,3205$ & $-0,0851$ & $-0,0465$ & 0,0791 & 0,4890 & 0,1697 & $-0,6286$ \\
$\varphi_{7}$ & 0,4675 & 0,3205 & $-0,0851$ & 0,0465 & 0,0791 & 0,4890 & 0,1697 & 0,6286 \\
$\varphi_{8}$ & 0,1459 & 0,0000 & 0,5536 & 0,0000 & $-0,2124$ & $-0,2675$ & 0,7453 & 0,0000 \\
\hline
\end{tabular}

Tab. 4. MO's (Eigenvektoren) für $\mathrm{C}_{3} \mathrm{O}_{2} \mathrm{~S}_{3}$.

14 R. Zahradnik, Electronic Structure of Heterocyclic Sulfur Compounds in: Advances in Heterocyclic Chemistry Vol. 5, S. 1, Academic Press, New York 1965.

15 J. Fabian, A. Mehlhorn, A. Bormann u. R. Mayer, Wissensch. Z. Techn. Univ. Dresden 14, 285 [1965].

16 Vgl. hierzu J. Fabian, A. Mehlhorn u. R. Mayer, Z. Chem. $5,22[1965]$
17 A. Streitwieser, Molecular Orbital Theory for Organic Chemists, Wiley, New York 1961.

${ }^{17}$ a Die Rechnung wurde hauptsächlich durchgeführt, um später vergleichende Betrachtungen mit entsprechenden Werten bei ähnlichen Molekülen anstellen zu können. 
ten die Eigenvektoren (hier HMO's) enthält, ist in Tab. 4 angegeben. Die Bedeutung der einzelnen $\varphi_{\mathrm{i}}$ (AO's), die in die verschiedenen HMO's zu den einzelnen Eigenwerten $x_{\mathrm{i}}$ eingehen, geht aus dem folgenden Diagramm hervor:

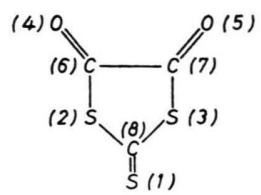

Als Gesamt- $\tau$-Elektronenenergie ergibt sich:

$$
E_{\pi}=16,6878 \beta \text {. }
$$

\section{Experimentelles}

$\mathrm{C}_{3} \mathrm{O}_{2} \mathrm{~S}_{3}$ wurde nach der Vorschrift von KREBS und Gatrow ${ }^{1}$ (vgl. auch l. c. ${ }^{18}$ ) dargestellt.
Die IR-Spektren wurden mit einem Leitz-IRSpektrographen im Bereich zwischen 4000 und $400 \mathrm{~cm}^{-1}$ gemessen. Es wurden Nujol-Suspensionen und Lösungen in $\mathrm{CCl}_{4}$ verwendet. Die Spektren der $\mathrm{CCl}_{4}$-Lösungen zeigten, abgesehen von einer nur äußerst schwach angedeuteten Aufspaltung der $v(\mathrm{CO})$-Bande, keine zusätzlichen Banden gegenüber den Nujol-Spektren.

Die Elektronen-Absorptionsspektren wurden mit einem PMQ II Zeiss-Gerät aufgenommen.

Die quantenchemischen Berechnungen wurden mit einer IBM 7040-Anlage durchgeführt.

Wir danken Herrn Prof. Dr. O. Glemser sehr für großzügige Unterstützung. R. A. dankt Herrn Prof. Dr. W. A. Bingel für großzügige Förderung. Der D e u t $\mathrm{schen}$ Forschungsgemeinschaf tanken wir für die zur Verfügung gestellten Hilfsmittel.

18 B. KREBS, Dissertation, Göttingen 1965.

\title{
Mittlere Schwingungsamplituden des Trithiocarbonat- und Triselenocarbonations
}

\author{
G. Nagarajan und A. Müller \\ Department of Chemistry, University of Maryland, College Park, Maryland, USA, und \\ Anorganisch-Chemisches Institut, Universität Göttingen \\ (Z. Naturforschg. 21 b, 393-396 [1966] ; eingegangen am 9. Dezember 1965)
}

\begin{abstract}
Mean-square amplitude quantities and mean amplitudes of vibration for $\mathrm{CS}_{3}{ }^{2} \ominus$ and $\mathrm{CSe}_{3}{ }^{2} \odot$ at $298{ }^{\circ} \mathrm{K}$ and $500^{\circ} \mathrm{K}$ are computed. The method of $\mathrm{Cyv}$ in utilizing symmetry coordinates has been employed. A brief discussion of the results is given.
\end{abstract}

In letzter Zeit ist verschiedentlich über IR- und Raman-spektroskopische Untersuchungen an kristallinen Trithiocarbonaten ${ }^{\mathbf{1}-5}$ und Triselenocarbonaten ${ }^{6}$ in der Literatur berichtet worden. Für das $\mathrm{CS}_{3}{ }^{2 \ominus}$-Ion sind außerdem Kraftkonstanten-Berechnungen (G.V.F.F. ${ }^{7,8}$ und U.B.F.F. ${ }^{9}$ ) durchgeführt worden. In der vorliegenden Arbeit werden mittlere Schwingungsamplituden für beide Ionen berechnet.

Die Reduktion der reduziblen Darstellung in der Basis kartesischer Verrückungsvektoren von ebenen

1 B. Krebs, G. Gattow u. A. Müller, Z. anorg. allg. Chem. 337, 279 [1965].

2 H. SeIdel, Naturwissenschaften 52, 257 [1965].

3 B. Krebs, A. Müller u. G. Gatrow, Z. Naturforschg. 20 b, 1017 [1965].

4 B. Krebs u. A. Müller, Z. Naturforschg. 20 a, 1664 [1965].

5 A. Müller u. M. Stockburger, Z. Naturforschg. 20 a, 1242 [1965].

6 H. SeIdel, Naturwissenschaften 52, 539 [1965].

7 B. Krebs u. A. Müller,Z. Naturforschg. 20 a, 1124 [1965].
$\mathrm{XY}_{3}$-Molekülen bzw. Ionen mit $\mathrm{D}_{3 \mathrm{~h}}$-Symmetrie ergibt (für die 6 eigentlichen Normalschwingungen)

$$
\Gamma_{\text {Schw }}=\mathrm{A}_{1}{ }^{\prime}(\mathrm{R})+\mathrm{A}_{2}{ }^{\prime \prime}(\mathrm{IR})+2 \mathrm{E}^{\prime}(\mathrm{R} ; \mathrm{IR}) .
$$

$v_{1}\left(\mathrm{~A}_{1}{ }^{\prime}\right)$ entspricht einer totalsymmetrischen $\mathrm{XY}$ Valenzschwingung, $v_{2}\left(\mathrm{~A}_{2}{ }^{\prime \prime}\right)$ einer nichtebenen Deformations-Schwingung, $v_{3}\left(\mathrm{E}^{\prime}\right)$ einer asymmetrischen XY-Valenzschwingung und $v_{4}\left(\mathrm{E}^{\prime}\right)$ einer ebenen asymmetrischen YX Y-Deformations - Schwingung. Eine schematische Darstellung der Normalschwingungen ist verschiedentlich angegeben worden ${ }^{10-12}$.

8 A. Fadini, A. Müller u. B. Krebs, Z. Naturforschg. 20 a, 1241 [1965].

9 A. Müller u. B. Krebs, Spectrochim. Acta [London], im Druck.

10 S. S. Silver u. W. H. Shaffer, J. chem. Physics 9, 599 [1941].

11 G. Herzberg, Infrared and Raman Spectra of Polyatomic Molecules, D. Van Nostrand Company, New York 1962.

12 T. Y. Wu,Vibration Spectra and Structure of Polyatomic Molecules, National University of Peking, Kun-Ming China, 1939. 